J. Clin. Chem. Clin. Biochem.

Vol. 15, 1977, pp. 705-708

\title{
Biochemisch-diagnostische Kriterien bei Glykogenose Typ II (Saure-Maltase-Defekt)
}

\author{
Von H. Pilz, H. H. Goebel, H. Stefan, D. Seidel und A. Kohlschütter \\ Neurologische Klinik, Neuropathologische Abteilung und Kinderklinik der Universität Göttingen, \\ Universitäts-Nervenklinik Bonn
}

(Eingegangen am 4. April/6. Juli 1977)

\begin{abstract}
Zusammenfassung: In Körperflüssigkeiten und Organgeweben (Biopsie- und Autopsiematerial) von Normalpersonen und zwei Patienten mit Typ-II-Glykogenose finden sich verschiedene Enzymmuster der sauren und neutralen $\alpha$-1,4-Glucosidase (Maltase). In Skeletmuskel, Herzmuskel, Leber und Hirnrinde eines Patienten mit der infantilen Krankheitsform (Pompe'sche Krạnkheit) ist die Aktivität der normalerweise überwiegenden sauren $\alpha$-1,4-Glucosidase stark vermindert, während die neutrale $\alpha-1,4-$ Glucosidase nicht betroffen ist. In der Niere des infantilen Patienten liegt eine deutliche, im Urin des Patienten mit adulter Krankheitsform eine partielle Verminderung beider Enzymformen vor; beide Komponenten weisen auch bei Normalpersonen eine etwa gleich hohe Aktivität auf. In den Leukocyten findet sich bei Pompe'scher Krankheit lediglich eine leichte Abnahme der auch normalerweise nur in

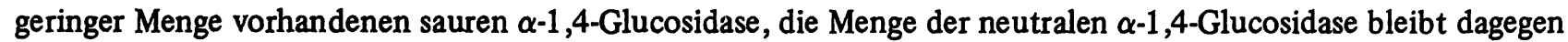
unverändert. Für eine "Screening"-Untersuchung bietet sich eine Messung der $\alpha$-Glucosidase im Urin an. Im Verdachtsfall erscheint eine eindeutige biochemische Abgrenzung des Krankheitsbildes nur durch die Bestimmung der sauren und neutralen $\alpha$-Glucosidase in bioptisch gewonnenem Muskel- und/oder Lebergewebe möglich.
\end{abstract}

\section{Biochemical diagnosis of glycogenosis type II (acid maltase deficiency)}

Summary: Body fluids and organ tissues (obtained at biopsy and/or autopsy) of controls and two patients with type-II glycogenosis showed different enzyme patterns of acid and neutral $\alpha$-1,4-glucosidase (maltase). The normally predominant acid $\alpha$-glucosidase was markedly deficient in skeletal muscle, cardiac muscle, liver and cerebral cortex of a patient with the infantile type of the disorder (Pompe's disease), while activity of the neutral $\alpha$-1,4-glucosidase was not decreased. In kidney and urine (the latter from a patient with the adult-onset type-II glycogenosis) a marked and a partial diminution of the acid and neutral $\alpha$-1,4-glucosidase respectively were found. Normal individuals also exhibit an approximately equal activity of both enzyme components. In leukocytes of the patient with Pompe's disease, there was only a slight decrease of the normally low acid $\alpha$-glucosidase activity, while the amount of the neutral enzyme was unchanged. For diagnostic screening, the determination of urinary $\alpha$-glucosidase is useful. But a final biochemical characterization of the metabolic disorder can only be performed by assay of acid and neutral $\alpha$-glucosidase in muscle and/or liver tissue obtained by biopsy.

\section{Einführung}

Bei dẹr Glykogenose vom Typ II handelt es sich um eine rezessiv vererbte Erkrankung, die durch eine progressive und fast generalisierte Glykogenspeicherung charakterisiert ist. Als Ursache der Glykogenspeicherung wurde ein Defekt der lysosomalen sauren Mältase gefunden. Diese spaltet vorwiegend 1,4- $\alpha$-glucosidische Bindungen, so daß dieses Enzym weitgehend mit der sauren $\alpha-1,4-$ Glucosidase (E.C. 3.2.1.20) identisch ist. Klinisch unterscheidet man infantile (Pompe'sche Krankheit), juvenile und adulte Manifestationsformen der Erkrankung. Das gemeinsame klinische Merkmal aller Verlaufsformen ist eine ausgeprägte Muskelschwäche. Bei den kindlichen Fällen kommen eine Cardiomegalie, leichte Hepatomegalie und gelegentlich bulbäre Symptome hinzu, während bei den Erwachsenenfallen die Myopathie im Vordergrund steht (1). Für den Neurologen ist von Bedeutung, daß bei allen ätiologisch unklaren Myopathien bzw. Muskeldystrophien differentialdiagnostisch eine Typ-II-Glykogenose in Erwägung gezogen werden muß (2). Die Diagnose wird in der Regel durch den licht- und elektronenmikroskopischen Nachweis der intralysosomalen Glykogenspeicherung in Muskel- 
und/oder Leber gestellt. Zusätzlich werden auch biochemische Untersuchungen vorgenommen.

Um die Wertigkeit der Enzymbefunde für diagnostische Zwecke darzulegen, haben wir die saure und neutrale $\alpha$-Glucosidase in Leukocyten, biopsiertem Organgewebe (Skeletmuskulatur, Leber) und Autopsiematerial (Herzmuskel, Gehirn, Niere) eines infantilen Falles sowie im Urin eines adulten Falles untersucht.

\section{Material und Methoden}

\section{Patienten und Untersuchungsmaterial}

Leukocyten, biopsiertes Organgewebe (Skeletmuskulatur, Leber) und Autopsiegewebe (Skeletmuskulatur, Herzmuskel, Leber, Niere, Großhirn) stammen von einem 9 Monate alten Knaben, der wie ein älteres Geschwister an klinisch, morphologisch und biochemisch gesicherter Pompe'scher Krankheit verstarb. Die Leukocyten wurden zu Lebzeiten des Patienten durch Dextransedimentation aus Venenblut gewonnen, Biopsieund Autopsiegewebe wurden nach Entnahme zusammen mit entsprechendem Normalgewebe sofort eingefroren $\left(-70^{\circ} \mathrm{C}\right)$. Gleichzeitig wurden Leukocyten eines altersentsprechenden Knaben ohne Hinweise für eine lysosomale Krankheit eingefroren. Weiteres Leukocytenmaterial stammt von gesunden Personen unterschiedlichen Alters.

Die klinischen und morphologischen Befunde des 40-jährigen Patienten mit Typ-II-Glykogenose wurden an anderer Stelle mitgeteilt (3). Der 24-Stunden-Urin dieses Mannes wurde zusammen mit einem Kontrollurin gekühlt transportiert, zentrifugiert und ebenfalls für wenige Tage tiefgefroren. Weitere Urinproben (24-Stunden-Menge) stammen von gesundem Laborpersonal, Studenten und gesunden Kindern.

\section{$\alpha$-Glucosidasebestimmung}

Der zentrifugierte und tiefgefrorene Urin wurde vor Gebrauch gegen dest. Wasser dialysiert und direkt zur Enzymbestimmung eingesetzt. Außerdem wurde der Kreatiningehalt im Urin be- stimmt. Leukocyten und Körpergewebe wurden ausgiebig manuell mit dest. Wasser im Verhältnis $1: 10(\mathrm{w} / \mathrm{v})$ homogenisiert (Mikro-Duall-Homogenisator), mehrfach eingefroren und wieder aufgetaut sowie zentrifugiert. Der Überstand entsprach der Enzymlösung. Der Enzymansatz für Leukocyten und Organgewebe setzte sich wie folgt zusammen (4): $10 \mu \mathrm{l}$ Enzymextrakt und $50 \mu \mathrm{l} 1 \mathrm{mmol} / 1$ 4-Methylumbelliferyl- $\alpha$-glucosid

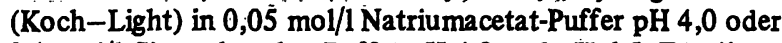
$0,1 \mathrm{~mol} / 1$ Citratphosphat-Puffer $\mathrm{pH} \mathrm{4,0}$ und $\mathrm{pH} 6,5$. Für die Erstellung der pH-Aktivitätskurven wurde Citratphosphat-

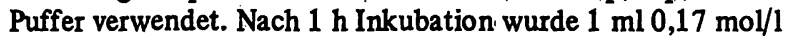
Glycincarbonat-Puffer $\mathrm{pH}$ 10,5 zugegeben und die durch das freigesetzte 4-Methylumbelliferon entstehende Fluöresżenz in einem Turner-Fotometer (Modell 111) gegen einen Ansatz ohne Enzym mit dem Absorptionsfilter Wratten 48 und $2 A(450 \mathrm{~nm})$ sowie dem Emmissionsfilter 7-60 (365 nm) gemessen. Als Standard diente freies 4-Methylumbelliferon (Koch-Light).

Der Enzymansatz für Urin hatte folgende Zusammensetzung: $50 \mu$ zentrifugierter und dialysierter Ürin, $300 \mu$ SubstratPuffer-Lösung, $3 \mathrm{ml} \mathrm{0,17} \mathrm{mol} / 1$ Glycincarbonat-Puffer pH 10,5.

Es wurden grundsätzlich Doppelbestimmungen durchgeführt.

\section{Ergebnisse}

Im Gewebe aller normalen untersuchten inneren Organe findet sich ein Überwiegen der sauren, lysosomalen $\alpha$-Glucosidase gegenüber der neutralen, nichtlysosomalen Enżymkomponente (Tab. 1). Eine Ausnahme bildet lediglich die Niere, bei der ebenso wie im Urin das Optimum der Hauptenzymaktivität etwas weiter zum neutralen Bereich, nämlich nach $\mathrm{pH} 5,0$, verschoben ist (Abb. 1). Daraus erklärt sich die Tatsache, daß die bei pH 4,0 und pH 6,5 gemessenen Enzymanteile etwa gleichgroß sind bzw. nur geringe Differenzen aufweisen (Tab. 1).

Wieder anders liegen die Verhältnisse in den Leukocyten deș normạlen Vergleichsfalles, wo die Aktivität der neu-

Tab. 1. Aktivität der sauren und neutralen $\alpha$-Glucosidase in Urin, Leukocyten sowie Biopsie- und Autopsiegeweben bei infantiler und adulter Glykogenose vom Typ II im Vergleich zu Kontrollfällen.

\begin{tabular}{|c|c|c|c|c|c|}
\hline & & \multicolumn{2}{|c|}{$p H 4,0$} & \multirow{2}{*}{$\begin{array}{l}\text { pH } 6,5 \\
\text { Phosphat-Puffer }\end{array}$} & \\
\hline & & Acetat-Puffer & Phosphat-Puffer & & \\
\hline \multicolumn{6}{|c|}{ Infantile Typ-II-Glykogenose } \\
\hline Leukocyten* & $\begin{array}{l}\text { Vergl.-Kontrolle*** } \\
\text { Weitere Kontrollen } \\
(\mathrm{n}=14), 5-62 \mathrm{~J} . \\
\text { Pat. F.A. }\end{array}$ & $\begin{array}{l}13,8 \\
29,5 \mp 10,8 \\
(13,5-46,8) \\
6,4\end{array}$ & 7,5 & 36,0 & . \\
\hline & $\begin{array}{l}\text { Kontrolle } \\
\text { Pat. F.A. }\end{array}$ & $\begin{array}{r}10,6 \\
0,3\end{array}$ & $\begin{array}{c}13,6 \\
0\end{array}$ & $\begin{array}{l}9,9 \\
5,4\end{array}$ & \\
\hline Herzmuskel* & $\begin{array}{l}\text { Kontrolle } \\
\text { Pat. F.A. }\end{array}$ & $\begin{array}{r}73,0 \\
0,5\end{array}$ & $\begin{array}{r}71,0 \\
0,8\end{array}$ & $\begin{array}{l}7,5 \\
3,4\end{array}$ & \\
\hline & $\begin{array}{l}\text { Kontrolle } \\
\text { Pat. F.A. }\end{array}$ & $\begin{array}{r}69,0 \\
0,2\end{array}$ & $\begin{array}{r}78,0 \\
0,1\end{array}$ & $\begin{array}{l}19,0 \\
18,1\end{array}$ & \\
\hline & $\begin{array}{l}\text { Kontrolle } \\
\text { Pat. F.A. }\end{array}$ & $\begin{array}{c}66,0 \\
0\end{array}$ & $\begin{array}{c}60,0 \\
0\end{array}$ & $\begin{array}{l}7,9 \\
7,2\end{array}$ & \\
\hline Niere* & Kontrolle & $\begin{array}{r}174,0 \\
0,1\end{array}$ & $\begin{array}{r}155,0 \\
0,3\end{array}$ & $\begin{array}{r}128,0 \\
0,8\end{array}$ & \\
\hline \multicolumn{2}{|c|}{ Adulte Typ-II-Glykogenose } & . & & & \\
\hline Urin** & $\begin{array}{l}\text { Vergl.-Kontrolle*** } \\
\text { Weitere Kontrollen } \\
(\mathrm{n}=5), 7-55 \mathrm{~J} . \\
\text { Pat. K.K. }\end{array}$ & $\begin{array}{l}117,1 \\
207,7 \mp 77,3 \\
(93,2-268,1) \\
20,9\end{array}$ & $\begin{array}{l}168,6 \\
\cdot 32,1\end{array}$ & 167,1 & \\
\hline
\end{tabular}

\footnotetext{
* als bei $37^{\circ} \mathrm{C}$ freigesetztes 4-Methylumbelliferon [nmol/1 $\cdot \mathrm{h} \cdot \mathrm{mg}$ Protein]

** als bei $37^{\circ} \mathrm{C}$ freigesetztes 4-Methylumbelliferon [nmol/1 $\cdot \mathrm{h} \cdot(\mathrm{mg}$ Kreatinin $/ 24 \mathrm{~h})$ ]

*** Gleiches Alter und Geschlecht, gleiche Transport- und Aufarbeitungsbedingungen
} 


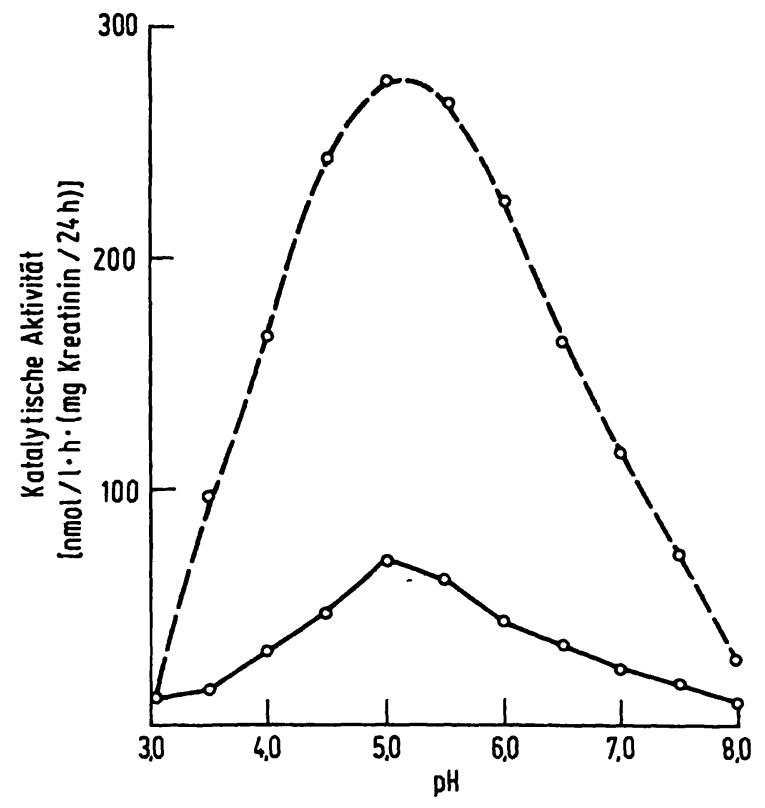

Abb. 1. pH-Aktivitäts-Kurve der $\alpha$-Glucosidase im Urin eines Patienten ( $0-0$ ) mit Typ-II-Glykogenose (adulte Form) im Vergleich zu einer entsprechenden Kontrolle (0--- 0$)$. Methodik s. Text.

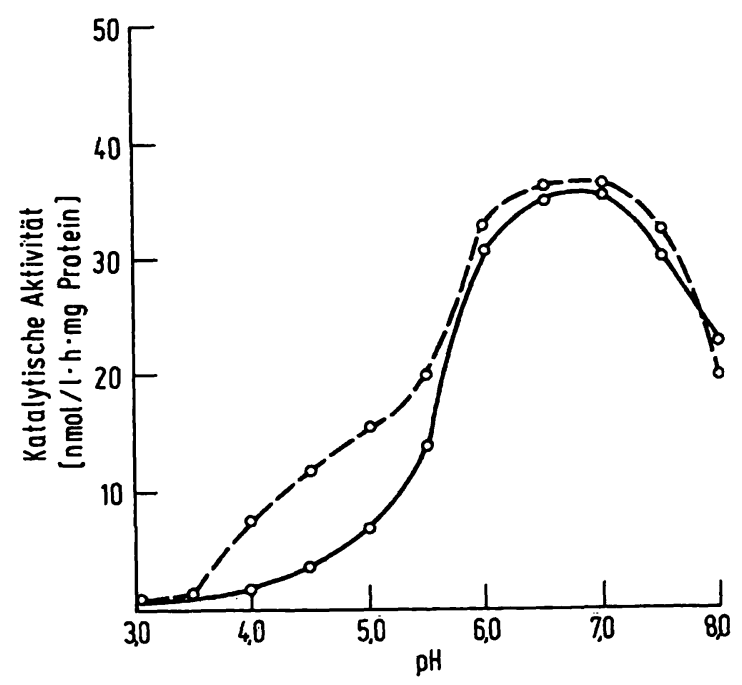

Abb. 2. pH-Aktivitäts-Kurve der $\alpha$-Glucosidase in den Leukocyten eines Patienten $\left(0_{-}-0\right)$ mit Typ-II-Glykogenose (infantile Form) im Vergleich zu einer entsprechenden Kontrolle (0-.-0). Methodik s. Text.

tralen $\alpha$-Glucosidase über derjenigen der sauren $\alpha$-Glucosidase liegt (Tab. 1; Abb. 2).

Entsprechend diesen unterschiedlichen Befunden in K̈örperflụ̈ssigkeiten und Organgeweben von Normalpersonen lassen sich auch quantitative und qualitative Unterschiede im Verhältnis vo on saurer zu neutraler $\alpha$ Glucosidase bei Patienten mit Typ-II-Glykogenose nachweisen. In Ŝkelet- und Herzmuskel, Leber sowie Gehirn des infantilen Patienten liegt ein hochgradiger Mangel der sauren $\alpha$-Glucosidase bei nicht oder nur wenig veränderter Aktivität der neutralen $\alpha$-Glucosidase vor
(Tab. 1). Auch in der Niere ist der Enzymdefekt stark ausgeprägt. Im Urin des adulten Patienten ist im Vergleich zu einem unter gleichen Bedingungen untersuchten Kontrollurin einer gleichaltrigen Person eine allgemeine Verminderung der Enzymaktivität vorhanden (Abb. 1), so daß im Urin, ähnlich wie in der Niere, eine Verminderung sowohl der sauren wie auch der neutralen $\alpha$-Glucosidase resultiert (Tab. 1). Allerdings ist die enzymatische Aktivität im Urin des adulten Patienten nicht so weit erniedrigt wie in den Organgeweben des infantilen Patienten. Die Verminderung der sauren $\alpha$-Glucosidase wird im Vergleich zu weiteren Kontrollbefunden eher noch deutlicher.

Die geringste Abweichung der Enzymaktivitäten von einer gleichgeschlechtlichen und altersentsprechenden Kontrolle findet sich in den Leukocyten des infantilen Falles, wo lediglich eine leichte Abnahme der Enzymaktivität bei pH 4,0 festzustellen ist, während bei pH 6,5 signifikante Differenzen nicht bestehen (Tab. 1; Abb. 2). Der normale Vergleichswert der sauren $\alpha$ Glucosidase liegt dabei im unteren Bereich von 14 weiteren gesunden Personen unterschiedlichen Alters und Geschlechts.

\section{Diskussion}

Sowohl die saure wie auch die neutrale Mal tase spalten das synthetische chromogene bzw. fluorogene Substrat$p$-Nitrophenyl- $\alpha$-glucosid bzw. 4Methylumbelliferyl$\alpha$-glucosid, so daß sie zur Messung dieser Enzymkomponenten eingesetzt werden können (1). Wegen der besonderen Empfindlichkeit haben wir letzteres für unsere Untersuchungen gewählt.

Wie die Ergebnisse zeigen, lassen sich in Körperflüssigkeiten und Organgeweben von Normalpersonen wie auch von Patienten mit Typ-II-Glykogenose verschiedene Enzymmuster der sauren und neutralen $\alpha$-Glucosidase unterscheiden.

Wir konnten bestätigen, daß in Skeletmuskel, Herzmuskel, Leber und Hirnrinde bei infantiler Typ-IIGlykogenose ein hochgradiger Defekt der sauren Maltase vorliegt, die normalerweise eine höhere Aktivität als die neutrale Maltase besitzt (5). Eine Korrelation zwischen dem kompletten Enzymdefekt in der Hirnrinde und den dort nachweisbaren morphologischen Veränderungen soll durch weitere Untersuchungen geklärt werden. Bisher wurde die stärkste Glykogenspeicherung im Hirnstamm beschrieben, während die Hirnrinde weniger betroffen sein soll (1). In der Niere sind nach unseren Befunden beide Glucosidasen vom Defekt betroffen. Andere Untersucher haben festgestellt, daß sich das Nierenenzym hinsichtlich seiner kinetischen und physikalisch-chemischen Eigenschaften deutlich von denjenigen anderer Organe unterscheidet. Dabei wurde nur eine partielle Verminderung der sauren $\alpha$-Glucosidase nachgewiesen (6). Die geringere Hitzestabilität der sauren 
$\alpha$-Glucosidase in der Niere von Patienten mit Pompescher Krankheit und das Fehlen einer positiven immunologischen Kreuzreaktion gegen Antiserum des Leberenzyms weisen darauf hin, daß es sich beim pathologischen Restenzym der Niere wahrscheinlich um eine spezielle Enzymform, die in den anderen Organen nicht vorkommt, handelt $(6,7)$.

Im Urin sind die quantitativen Verhältnisse nicht so eindeutig wie in der Niere, da zwar auch beide Enzymformen betroffen, aber nur partiell vermindert sind. Zwischen $\mathrm{pH}$ 4,5 und 5,5 ist im normalen Urin der altersentsprechenden Vergleichsperson ein hoher Aktivitätsgipfel vorhanden. Auch andere Untersucher haben darauf hingewiesen, daß im Urin das pH-Optimum der Maltase etwas mehr in den neutralen Bereich verschoben ist und schlossen daraus auf das Vorkommen einer dritten Enzymkomponente. Allerdings haben wir im Gegensatz zu diesen Autoren $(8,9)$ keine signifikanten Differenzen im pH-Optimum zwischen Patienten- und Kontrollurin gefunden. Urin als Enzymquelle wird auch zur Diagnose anderer lysosomaler Speicherkrankheiten, wie z.B. der $\mathrm{G}_{\mathrm{M} 2}$ - und $\mathrm{G}_{\mathrm{M} 1}$-Gangliosidose, der metachromatischen Leukodystrophie und des MaroteauxLamy-Syndroms herangezogen $(4,10,11,12)$. Zwar schwanken beim Bezug der Enzymaktivitäten auf die Urinmenge wegen der unterschiedlichen Urinausscheidung die gemessenen Werte oft erheblich. Von uns zur Zeit durchgeführte systematische Untersuchungen mit markierten und synthetischen Substraten verschiedener lysosomaler Hydrolasen scheinen jedoch zu zeigen, daß unter Berücksichtigung der Kreatininausscheidung innerhalb von 24 Stunden (Clearance-Funktion der Niere) wesentlich bessere Resultate erzielt werden (s. auch 1.c. (13)). Ob eine Altersabhängigkeit der mit dem Urin ausgeschiedenen $\alpha$-Glucosidase-Aktivität vorliegt, kann wegen der noch geringen Anzahl an kindlichen Urinproben bis jetzt nicht eindeutig entschieden werden. Die Enzymmenge des undialysierten Urins nimmt bei Raumtemperatur innerhalb von einer Woche nur um etwa $10 \% \mathrm{ab}$. Möglicherweise ist die im unteren Norm- bereich der übrigen Kontrollgruppe liegende Enzymaktivität des unter gleichartigen Bedingungen bestimmten Urins der altersentsprechenden Kontrollperson auf die Transportbedingungen zurückzuführen.

Eine dritte Art von Enzymspektrum ist auf Grund unserer Messungen in den Leukocyten vorhanden. Die bis jetzt hierzu in der Literatur mitgeteilten Befunde sind sehr widersprüchlich. Einige Untersucher wiesen eine mehr oder weniger ausgeprägte Enzymverminderung (saure $\alpha$-Glucosidase) in den Leukocyten nach, während andere überhaupt keinen Defekt fanden, was besonders für die adulte Verlaufsform gelten soll $(14,15,16,17)$. Wahrscheinlich werden diese differierenden Befunde durch eine lediglich leichte Abnahme der schon normalerweise nur in geringer Menge vorhandenen leukocytären sauren $\alpha$-Glucosidase erklärt, wie sie bei unserem Patienten mit Pompe'scher Krankheit vorliegt. An Hand der $\mathrm{pH}$-Aktivitäts-Kurven läßt sịch nämlich demonstrieren, daß sich ein geringfuigiger Defekt der leukocytären sauren Maltase nur in einem steileren Anstieg der Enzymkurve äußert. Auch unter Berücksichtigung der für das Ürinenzym gemachten Einschränkungen sind Leukocyten für diagnostische Zwecke offenbar weniger geeignet als Urin. Bei klinischen Verdachtsfällen sollten aber trotzdem für ein erstes „Screening“ Bestimmungen der $\alpha$-Glucosidase in Leukocyten und Urin durchgefuihrt werden. Eine eindeutige biochemische Diagnose des sauren Maltase-Mangel-Syndroms läßt sich unseres Erachtens jedoch nur.durch die Messung der sauren und neutralen $\alpha$-Glucosidase in bioptisch gewonnenem Muskel- oder Lebergewebe im Vergleich zu entsprechendem Normalgewebe stellen.

\section{Danksagung}

Wir sind Frau Rita Oberschmidt und Frau Hannelore Knop für die Durchführung der Enzymmessungen, Herrn Dr. Rühl vom Institut für klinische Pathologie des Stadtkrankenhauses Wolfsburg für die Überlassung des Sektionsgutes sehr dankbar. Die Untersuchungen wurden finanziell von der Deutschen Forschungsgemeinschaft (SFB 33) unterstütżt.

\section{Literatur}

1. Hers, H. G. \& de Barsy, T. (1973), Lysosomes and Storage Diseases (Hers, H. G. \& van Hoof, F., eds.) Academic Press, New York, S. 197-216.

2. Engel, A. G. (1970), Brain 93, 599-616.

3. Gullotta, F., Stefan, H. \& Mattern, H. (1976), J. Neurol. 213, 199-216.

4. Pilz, H., Heipertz, R. \& Seidel, D. (1977), Fortschr. Neurol. Psychiatr. (im Druck).

5. Steinitz, K. \& Rutenberg, A. (1967), Isr. J. Med. Sci. 3, 411-421.

6. Salafsky, I. S. \& Nadler, H. L. (1973), J. Lab. Clin. Med. 81, 450-454.

7. Koster, J. F., Slee, R. G., van der Klei-van Moorsel; J. M., Rietra, P. J. G. M. \& Lucas, C. J. (1976), Clin. Chim. Acta $68,49-58$

8. Mehler, M. \& di Mauro, S. (1976), Arch. Neurol. (Chicago) $33,692-695$.

9. Salafsky, I. S. \& Nadier, H. L. (1973), J. Ped. 82, 294-297.

10. Thomas, G. H. (1969), J. Lab. Clin. Med. 74, 725-731.

11. Saifer, A., Amoroso, J. \& Perle, G. (1976), Clin. Chim. Acta 67, 315-319.

12. Austin, J. H., Armstrong, D., Shearer, L. \& McAfee, D. (1966), Arch. Neurol. (Chicago) 14, 259-269.

13. Raab, W. P. (1972), Clin. Chem. 18, 5-25.

14. Illingworth-Brown, B. \& Zellweger, H. (1966), Biochem. J. $101,16 \mathrm{c}-18 \mathrm{c}$.

15. Koster, J. F., Slee, R. G. \& Hülsmann, W. C. (1974), Clin. Chim. Acta $51,319-325$.

16. Martin, J. J., de Barsy, T. \& den Tandt, W. R. (1976), J. Neurol. 213, 105-118.

17. Seiler, D., Kelleter, R. \& Kölmel, H. W. (1973), Experientia 29, 972-973.

Prof. Dr. H. Pilz

Neurologische Universitätsklinik und Poliklinik Martinistraße 52

D-2000 Hamburg 20 